\title{
Spectrophotometric Determination of Thiamine hydrochloride Via Oxidative Coupling Reaction Using 4-Aminoantipyrine
}

\author{
Reem A. Al-Luhaiby ${ }^{1 *}$, Mohammed S. Al-Enizzi ${ }^{2}$ \\ ${ }^{1,2}$ Department of Chemistry, College of Education for Girls, University of Mosul, Mosul, Iraq \\ E-mail: ${ }^{1 *}$ remawy92@gmail.com, ${ }^{2}$ mohammedsalim1977@yahoo.com
}

(Received April 08, 2020; Accepted May 30, 2020; Available online December 01, 2020)

DOI: 10.33899/edusj.2020.126895.1062, @ 2020, College of Education for Pure Science, University of Mosul.

This is an open access article under the CC BY 4.0 license (http://creativecommons.org/licenses/by/4.0/).

\begin{abstract}
:
A sensitive spectrophotometric method has been developed for the determination of thiamine hydrochloride by oxidative coupling reaction of thiamine with the reagent 4-aminoantipyrine (4AAP) in the presence of copper sulphate as oxidizing agent in alkaine medium forming a reddish brown colour. The product show maximum absorption at $335 \mathrm{~nm}$. The molar absorptivitiy is $26410.591 / \mathrm{mol} . \mathrm{cm}$ for concentrations obeyed Beer's law in the range $0.2-18 \mu \mathrm{g} \cdot \mathrm{ml}^{-1}$. The recovery was $100.93 \%$ with relative standard deviation $<2.0 \%$ for thiamine hydrochloride.Thiamine and reagent 4 -AAP product was formed in the ratio of $1: 1$. The stability constant of the product was $3.39 \times 10^{6} 1 . \mathrm{mol}^{-1}$ for thiamine hydrochloride indicating the good stability of this product. The optimum conditions for full colour development are described and the proposed method was applied successfully for determination of thiamine in the pharmaceutical preparation (Neurorubine). The common excipients used as additives in pharmaceutical do not interfere in the proposed method.
\end{abstract}

Key words: Oxidative coupling; Spectrophotometry; Thiamine hydrochloride; 4-aminoantipyrine

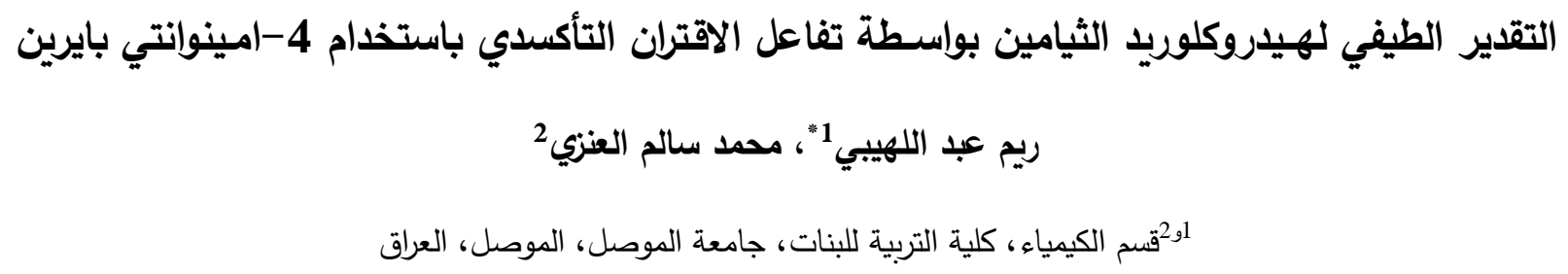

الخلاصة:

تـم تطـوير طريقة طيفيـة حساسة لتقدير هيدروكلوريد الثيامين عن طريق الاقتران التأكسدي للثيامين مـع الكاشف 4-

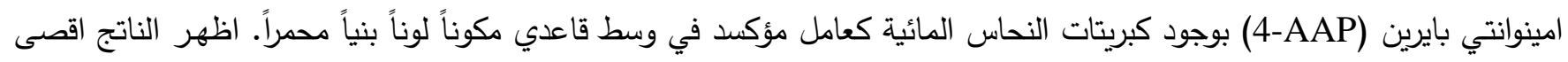

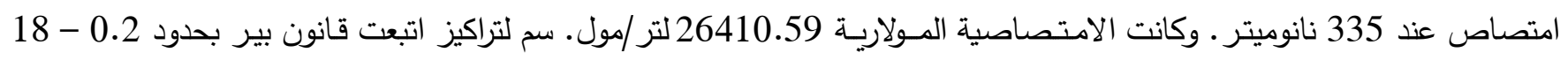

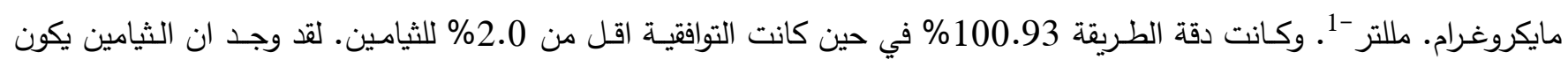

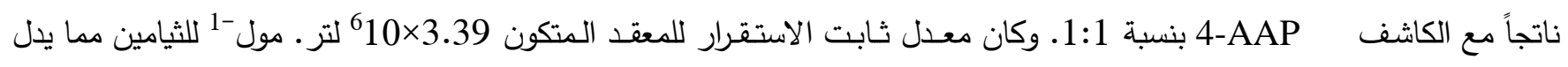
على الاستقرار الجيد لذلك الناتج. تمت دراسـة الظروف المثلى للتفاعل وطبقت الطريقة بنجاح لتقديـر الثيامين في المستحضر 
الصيدلاني (Neurorubine)، كما بينت النتـائج عـدم حدوث تداخل في الطــريقة المطـورة من قبـل المـواد الســواغ بـوصفهـا مضــافـات في المستــ حضـر ات الـصيدلانية. الكلمات المفتاحية: الأقتران التأكسدي، مطياف فوتومتري، هيدروكلوريد الثيامين، 4-امينوانتي بايرين

هيدروكلوريد الثيامين هو مركب دوائي له القابلية على الذوبان في الماء، يوجد في الأطعمة كالحبوب واللحوم والمكسرات والفول

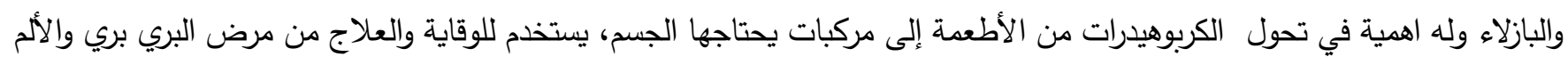
العصبي. ويؤدي نقصه الى الثعور بالضيق والإرتباك وفقدان الوزن كما يمكن أن يؤدي للوفاة اذا لم يعالج(1). ويمتلك هيدروكلوريد الثيامين

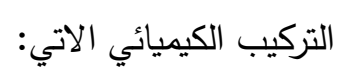<smiles>Cc1ncc(C[n+]2csc(CCO)c2C)c(N)n1</smiles>

3- [(4- amino-2 - methyl-5 - pyrimidinyl)methyl]- 5-(2-hydroxyethyl)- 4- methylthiazolium [1], hydrochloride M.wt $=337.3$ gm . mol $^{-1}$

وقد تم استخدام عدة طرق تحليلية في تقدير الثيامين منها الطرق الطيفيـة(2-11)، والكروماتوغرافيـة(12-15، والكهربائيـة(16).

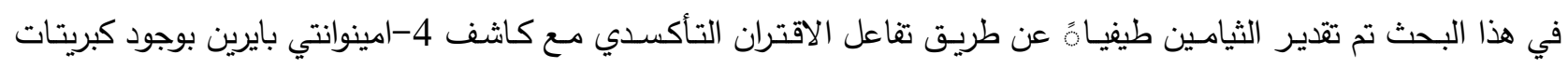
النحاس كعامل مؤكسد وفي وسط قاعدي وتم تطبيق الطريقـة بنجاح على المستحضر الصيدلاني (Neurorubine).

\section{الجزء الـعلي Experimental part}

Apparatus used الأجهزة المستخدمة

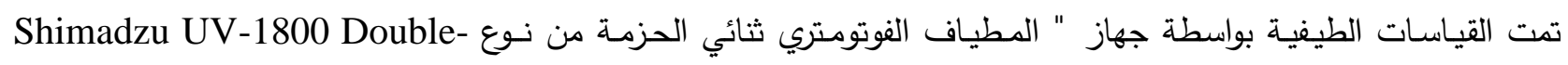

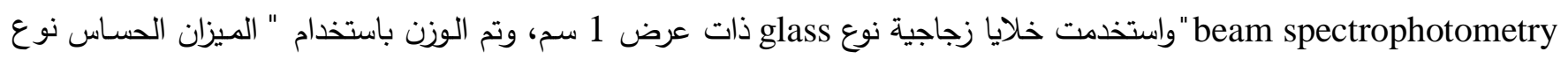

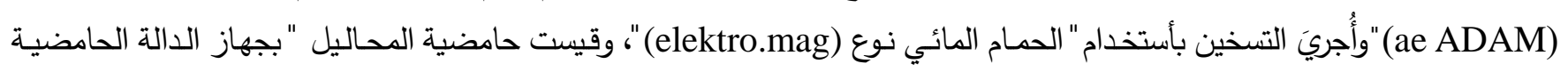

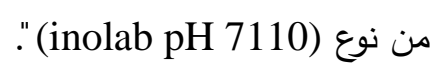
محاليل المواد الكيميائيـة

كانت جميع المواد الكيميائية المستخدمة على درجة عالية من النقاوة. - هيدروكلوريـــ الثيامين (100 جزء لكل مليون) حضر بإذابة 0.010 غم من المادة النقيـة في الماء المُقطر ثم نقل الى قنينة حجمية سعة 100 مللتر . - 4-امينوانتي بايرين (1\%) حضر بإذابة 1.0 غرام من المادة النقية في الماء المقطر ثم نُقل الى قنينة حجمية سعة 100 ملتر 


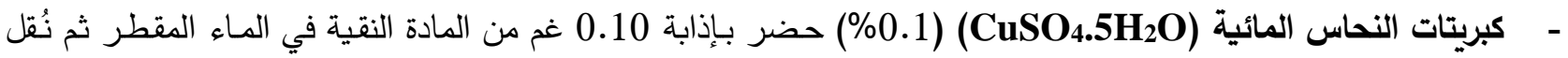
الى قنينة حمية سعة 100 مللتر. - - هيدروكسيد الصوديوم (5.0×10-20 مولاري) حضر بإذابة 0.50 غم من المادة النقية في الماء المقطر ثم نقل الى قنينة حجمية سعة 250 مللتر. النتائج والمناقثة

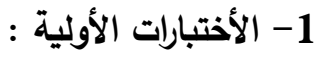
عند إضافة 1.0 مللتر من 4-امينوانتي بايرين بتركيز 1\% الى 1.0 مللتر من الثيامين وبتركيز 100 مايكروغراج/ مللتر ، ثم الثماكي

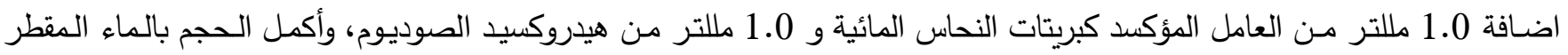
الى 25 مللتر وترك المحلول لمدة 10 دقائق في درجـة حرارة المختبر (30 من )، وقيس طيف الامتصاص للمحلول البني المحمر الناتج ووجد ان اعلى امتصاص يصل اليه المركب الدوائي عند الطول الموجي 335 نانوميتر . 2- 2 - 2 دراسة ظروف التفاعل المثلى درس تأثير عدة متغيرات في شدة الامتصاص للمعقد المتكون بغية الحصول على الظروف المثالية للتفاعل بين الثيامين والكاشف 4-امينوانتي بايرين، اذ أجريت الدراسات التالية بأستخدام 4.0 مايكروغراج/ مللتر من الثيامين.

\section{4-AAP دراسة كمية الكاشف}

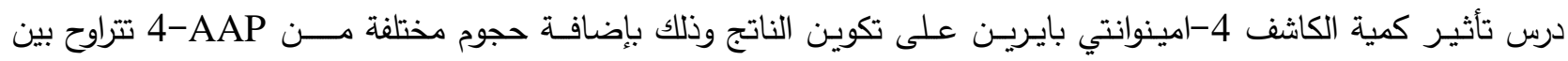
(2.0-0.0) مللتر تركيزه 1\% على الثيامين و1.0 مللتر من كبريتات النحاس المائية تركيز (0.1\%) و و1.0 مللتر من هيدروكسيد

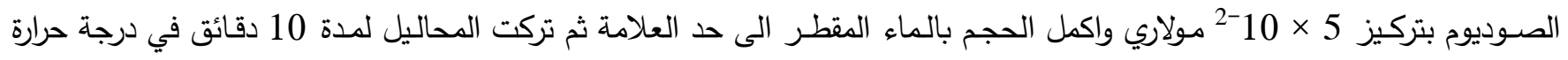

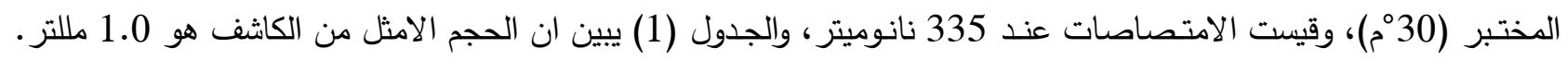
الجدول (1) كميـة الكاشف 4-امينوانتي بايرين

\begin{tabular}{|c|c|c|c|c|c|c|c|}
\hline $\begin{array}{c}\text { MI of 4-AAP } \\
\text { (1\%) }\end{array}$ & Without & 0.25 & 0.5 & 0.75 & 1.0 & 1.5 & 2.0 \\
\hline Absorbance & 0.11 & 0.179 & 0.203 & 0.237 & 0.316 & 0.302 & 0.143 \\
\hline
\end{tabular}

دراسة العامل المؤكسد

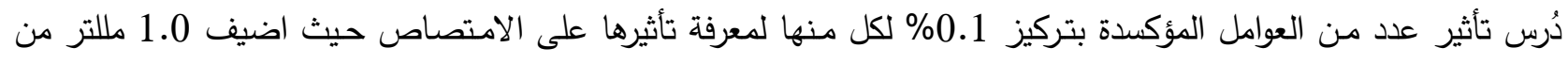

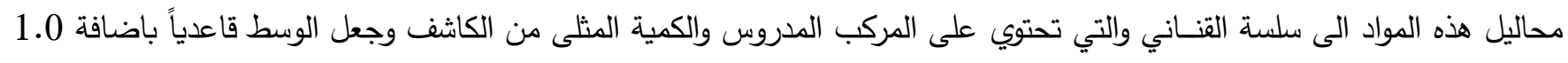

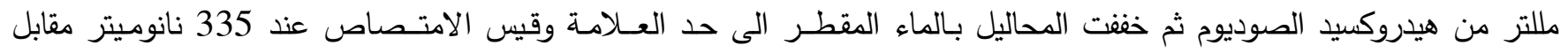

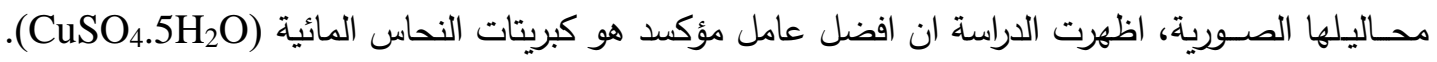




\begin{tabular}{|c|c|}
\hline \multicolumn{2}{|c|}{ الجدول (2) تأثير نـوع العـامل المؤكسد في شدة امتصاص الناتج| } \\
\hline Oxidizing agent $\mathbf{0 . 1 \%}$ & Absorbance \\
\hline Copper Sulphate.5H2O & 0.316 \\
\hline N- Bromosuccinimide & 0.005 \\
\hline Potassium Dichromate & 0.118 \\
\hline Potassium Iodate & 0.108 \\
\hline Sodium nitroprusside & 0.121 \\
\hline Potassium Periodate & 0.095 \\
\hline Potassium hexacyano ferrate(III) & 0.125 \\
\hline
\end{tabular}

دراسة حجم العامل المؤكسد

درس افضل حجم للعامل المؤكسد كبريتات النحاس المائية وذلك باستعمال حجوم متزايدة من العامل المؤكسد (0.0-3.0) مللتر ، الجدول (3) يبين الحجم الامثل من العامل المؤكسد.

الجدول (3) دراسة حجم العامل المؤكسد

\begin{tabular}{|c|c|c|c|c|c|c|c|}
\hline $\begin{array}{c}\text { Ml of 0.1\% } \\
\text { CuSO4.5H2 }\end{array}$ & Without & 0.5 & 1.0 & 1.5 & 2.0 & 2.5 & 3.0 \\
\hline Absorbance & 0.044 & 0.259 & 0.316 & 0.329 & 0.343 & 0.300 & 0.291 \\
\hline
\end{tabular}

من اجل اختيار القاعدة المناسبة للحصول على اعلى حساسية حضرت محاليل تحتوي على تراكيز متساوية من هيدروكلوريد الثيامين (4.0 مايكروغرام/ مللتر) واضيف 1.0 مللتر من الكاشف و2.0 ملتر من كبريتات النحاس المائية و.1.0 ملتتر مـن محاليل القواعد المختلفة ذات تركيز 5×10-2 مولاري وتم تخفيف المحاليل بالماء المقطر الى حد العلامسـة وقياس الامتصاص عند عند 335 نانوميتر مقابل المحلول الصوري، الجدول (4) يوضح النتائج التي تم التوصل اليها. الجدول (4) تأثير نوع القواعد

\begin{tabular}{|c|c|c|c|c|}
\hline Base $\left(\mathbf{5} \times \mathbf{1 0}^{-2} \mathbf{M}\right)$ & $\mathrm{NaOH}$ & $\mathrm{KOH}$ & $\mathrm{NH}_{4} \mathrm{OH}$ & $\mathrm{Na}_{2} \mathrm{CO}_{3}$ \\
\hline Absorbance & 0.343 & 0.272 & 0.110 & 0.115 \\
\hline
\end{tabular}

تأثير حجم القاعدة والدالة الحامضية

تمت دراسة افضل حجم من هيدروكسيد الصوديوم بتركيز (0.05 مولاري) وقياس pH المحاليل، ويبين الجدول (5) الدالة الهيدروجينيـة المثلى والحجم الامثل في التقدير وتم استخدمه في التجارب اللاحقة. 
الجدول (5) تأثير كمية القاعدة

\begin{tabular}{|c|c|c|c|c|c|c|}
\hline $\begin{array}{c}\text { Volume of NaOH } \\
\mathbf{5 \times 1 \mathbf { 1 0 } ^ { - 2 }} \mathbf{~ M ~ ( m l ) ~}\end{array}$ & Without & 0.5 & 0.75 & 1.0 & 1.5 & 2.0 \\
\hline Absorbance & 0.012 & 0.122 & 0.234 & 0.343 & 0.243 & 0.217 \\
\hline Final pH & 6.4 & 9.6 & 10.4 & 10.7 & 10.10 & 11.3 \\
\hline
\end{tabular}

ومن الجدول السابق يتبين ان 1.0 مللتر من القاعدة اعطى اعلى امتصاص عند دالة حامضية 10.7 لذا اعتبر الحجم الامثل كما درس تأثير (1.0 مللتر) من حامض الهيدروكلوريك بتركيز 5×10-2 مولاري على امتصاص المعقد الناتج ووجد بأن اضافته يسبب نقصاناً في شدة الامتصاص لذا تم استبعاد اضافة الحامض. تـأثير المحساليل المنظمة

$$
\begin{aligned}
& \text { درس تأثير عدة انواع من المحاليل المنظمة بدالة هيدروجينية } 10.7 \text { في امتصاص معقد الثيامين - - 20 - } \\
& \text { بايرين، ويوضح الجدول الاتي النتائج التي تم التوصل اليها. }
\end{aligned}
$$

الجدول (6) تأثير انواع المحاليل المنطمة في امتصاص المعقد

\begin{tabular}{|c|c|}
\hline Buffer solution pH 10.7 & Absorbance \\
\hline Without buffer (in presence of $\mathrm{N}_{\mathrm{a}} \mathrm{OH}$ ) & 0.343 \\
\hline $\mathrm{Na}_{2} \mathrm{~B}_{4} \mathrm{O}_{7} .10 \mathrm{H}_{2} \mathrm{O}+\mathrm{NaOH}$ & 0.253 \\
\hline $\mathrm{Na}_{2} \mathrm{CO}_{3}+\mathrm{NaHCO}$ & 0.262 \\
\hline $\mathrm{NaHCO}_{3}+\mathrm{NaOH}$ & 0.287 \\
\hline
\end{tabular}

تبين النتائج في الجدول اعلاه ان استخدام المحاليل المنظمة يؤدي الى نقصان في امتصاص المعقد لذا تم استبعادها في التجارب اللاحقة. تأثثير درجـة الحرارة والزمن

درس تأثير درجة الحرارة وزمن استقرار النواتج عند درجـات حرارة (30-60٪م) بـوجود كمية ثابتة من الثيامين 4.0 مايكروغرام/ مللتر والكميات المثلى من الكاشف والعامل المؤكسد والقاعدة ثم اكمال الحجم الى حد العلامة بالماء المقطر وقياس الامتصاص عند 335 نانوميتر ، يثير الثكل (1) ان المعقد يتكون بأعلى حساسية بعد 25 دقيقة من الاضافات عند 30ْم (درجة حرارة المختبر) ويبقى مستقراً اكثر من 50 دقيقة. 


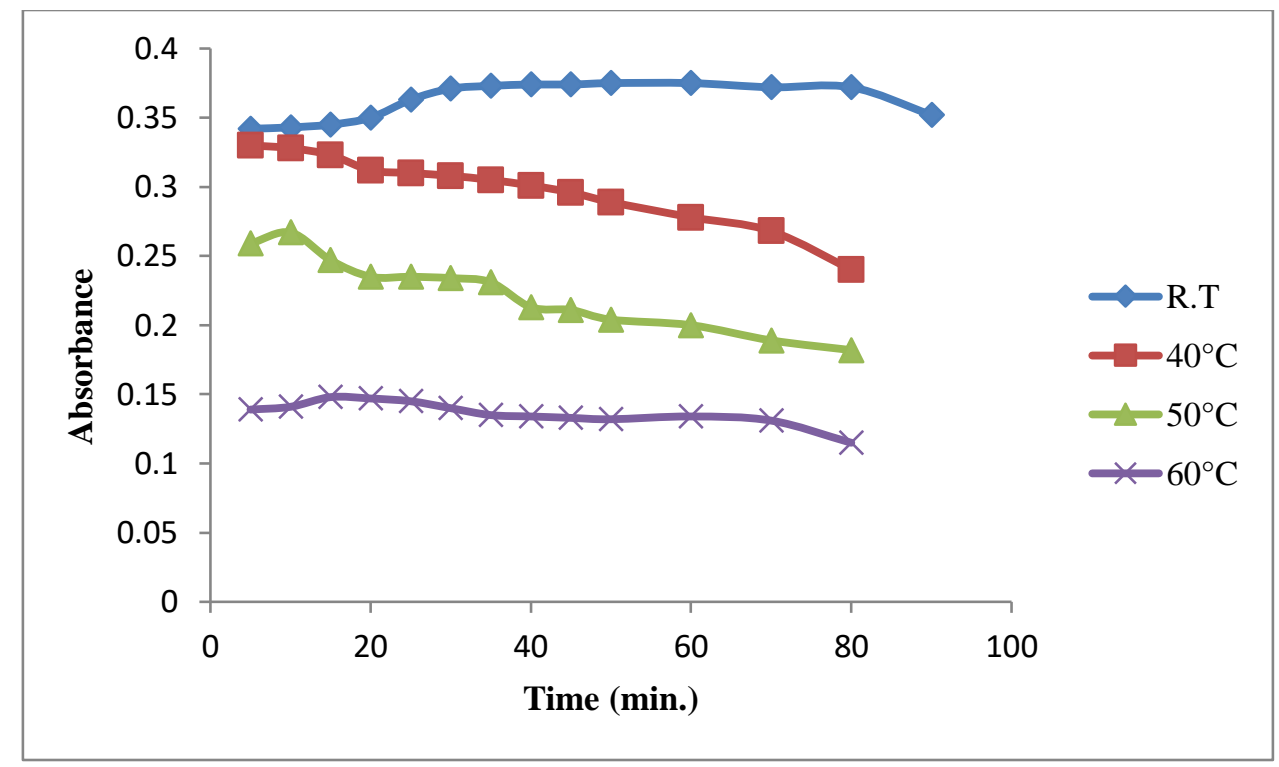

الشكل (1) دراسة درجـة الـحرارة وزمسن الاسـتقرار

دراسة تأثير مواد الثد السطحي

لغرض زيادة الحساسية درس تأثير عدد من مواد الثد السطحي ووجد بان تأثيرها سلبي على قيم الامتصـاص لذلك أستُبعدت من الدراسات الـلاحقة. تأثير تسلسل الاضافة درس تأثير تسلسل الاضافة على قيم امتصاص معقد الثيامين - 4-امينوانتي بايرين بإضافات متغيرة الترتيب للمركب الدوائي (R) والكاشف (R) (R) والعل المؤكسد) والقاعدة (B) مع مراعاة الظروف المثلى اللازمة لها، الجدول (7) يبين ان اضافة المحاليل بالتسلسل I والذي كان متبعاً في التجارب السابقة يعطي اعلى امتصاص للناتج.

الجدول (7) تأثير تسلسل الاضافة في تقدير هيدروكلوريد الثيامين

\begin{tabular}{|c|c|c|}
\hline Order Number & Reaction component & Absorbance \\
\hline I & $\mathrm{S}+\mathrm{R}+\mathrm{O}+\mathrm{B}$ & 0.364 \\
\hline II & $\mathrm{S}+\mathrm{O}+\mathrm{B}+\mathrm{R}$ & 0.330 \\
\hline III & $\mathrm{S}+\mathrm{B}+\mathrm{R}+\mathrm{O}$ & 0.361 \\
\hline IV & $\mathrm{S}+\mathrm{O}+\mathrm{R}+\mathrm{B}$ & 0.290 \\
\hline V & $\mathrm{R}+\mathrm{O}+\mathrm{B}+\mathrm{S}$ & 0.297 \\
\hline VI & $\mathrm{R}+\mathrm{B}+\mathrm{O}+\mathrm{S}$ & 0.211 \\
\hline VII & $\mathrm{R}+\mathrm{O}+\mathrm{S}+\mathrm{B}$ & 0.332 \\
\hline
\end{tabular}




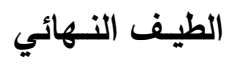

تم رسم الطيف النهائي لناتج الثيامين مـع (4-AAP) عند اطوال موجية 310-400 نانوميتر • لقد وجد ان اقصى امتصاص للناتج عند 335 نانوميتر ، الثكل (2) يبين الرسم النهائي للثيامين.

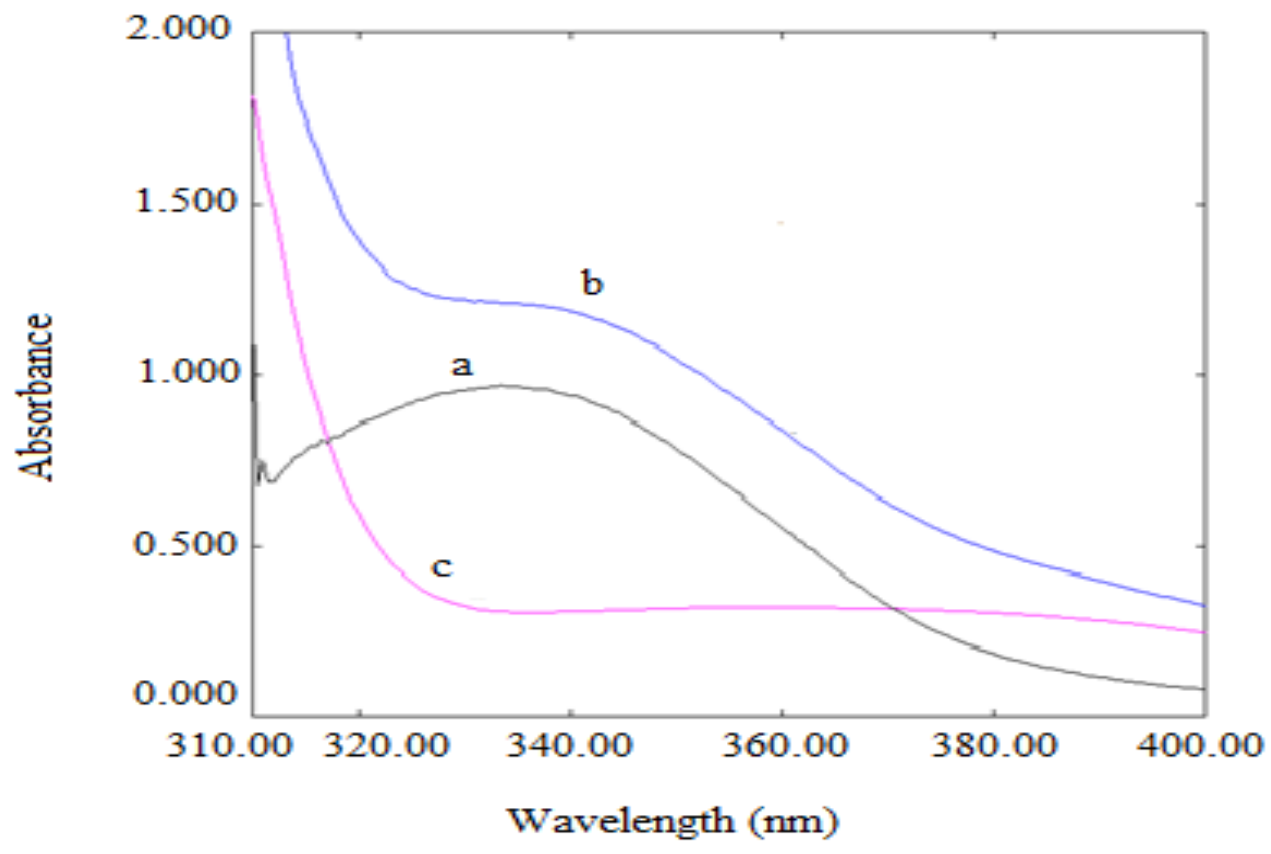

الثكل (2) طيف الامتصاص النهائي

a: الناتج الملون للثيامين (12 مايكروغرام. مللتر -1) مقابل المحلول الصوري b: الناتج الملون للثيامين مقابل الماء المقطر c: المحلول الصوري مقابل الماء المقطر دراسة الـمنحنى الـقياسي الى مجموعة قناني حجمية سعة 25 مللتر تمت اضافة كميات تتراوح بين 0.05-4.5 ملتر من محلول 100 مايكروغرام. مللتر - 100 ملترات 1 من هيدروكلوريد الثيامين تتبعه اضافة 1.0 مللتر من محلول 4-امينوانتي بايرين (1\%) و2.0 مللتر من محلول كبريتات النحاس المائية

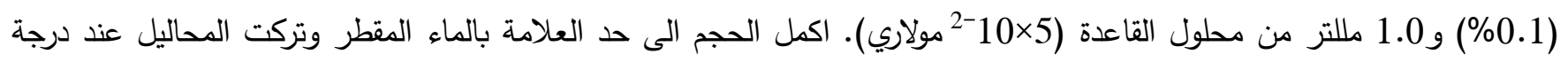
حرارة المختبر (30م) لمدة 25 دقيقة، وتم قياس الامتصاص مقابل المحلول الصوري عند الطول الموجي 335 نانوميتر .والثكل (3) يوضح المنحنى القياسي الذي يطيع قانون بير ضمن المدى (0.2 - 18) مايكروغرام/ مللتر ، بلـت قيمة معامل الامتصاص المولاري 26410.59 لتر / مـول • سـ في حين بلغ حد الكثف 0.229 مايكروغرام/ مللتر والحد الكمي 0.766 مايكروغرام/ مللتر . 


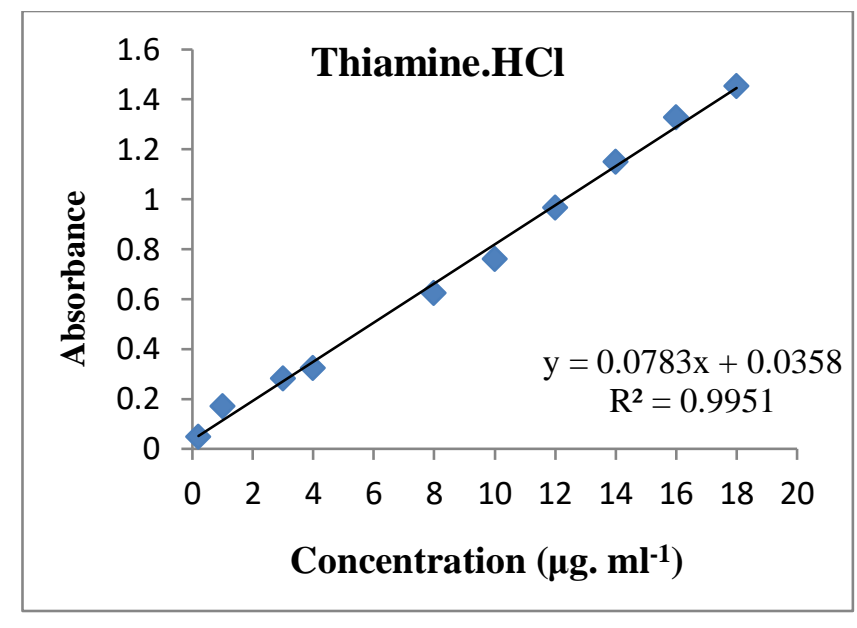

الثكل (3) المنحنى القياسي لتقدير هيدروكلـوريد الثيامين

الجدول (8) المواصفات الخطية والمدى التقديري والامتصاصية المولارية وحدود الكثف والتقدير الكمي في تقدير هيدروكلوريد الثيامين

\begin{tabular}{|c|c|c|c|c|c|c|c|}
\hline Compound & $\begin{array}{l}\text { Linearity } \\
\text { range } \\
\left(\mu \mathrm{g} \cdot \mathrm{ml}^{-1}\right)\end{array}$ & 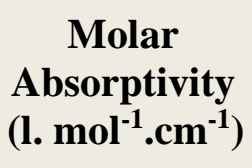 & $\begin{array}{c}\text { LOD }^{*} \\
\left(\mu \mathrm{g} \cdot \mathrm{ml}^{-1}\right)\end{array}$ & $\begin{array}{c}\text { LOQ* } \\
\left(\mu \mathrm{g} \cdot \mathrm{ml}^{-1}\right)\end{array}$ & Slope & Intercept & $\begin{array}{c}\text { Correlation } \\
\text { coefficient } \\
\left(\mathbf{R}^{\mathbf{2}}\right)\end{array}$ \\
\hline $\begin{array}{c}\text { Thiamine } \\
\text { hydrochloride }\end{array}$ & $0.2-18$ & 26410.59 & 0.229 & 0.766 & 0.0783 & 0.0358 & 0.9951 \\
\hline
\end{tabular}

*Average of ten determinations

دقة الطريقة وتـوافقها

تم حساب الدقة والتوافقية من خلال احتساب Recovery و RSD بأستخدام خمس قراءات لثلاثة تراكيز مختلفة، بلغ

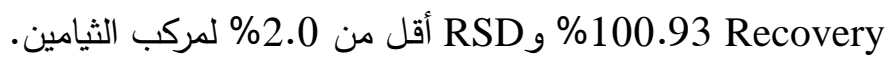

الجدول (9) دقة الطريقة وتـوافقها

\begin{tabular}{|c|c|c|c|c|}
\hline Compound & $\begin{array}{c}\text { Amount added } \\
\left(\boldsymbol{\mu g} \cdot \mathbf{~ m l}^{-\mathbf{1}}\right)\end{array}$ & $\begin{array}{c}\text { Recovery* } \\
(\boldsymbol{\%})\end{array}$ & $\begin{array}{c}\text { Average } \\
\text { recovery } \\
(\boldsymbol{\%})\end{array}$ & $\begin{array}{c}\text { RSD* } \\
(\mathbf{\%})\end{array}$ \\
\hline Thiamine & 1 & 102.00 & & 1.91 \\
hydrochloride & 4 & 99.60 & 100.93 & 0.98 \\
\hline
\end{tabular}

*Average of Five Determinations. 


\section{تأثير طبيعة الـمقد وثابت استقراربته}

اتبعت " "طريقة التغير المستمر Job's Method "(17) و " النسب المولية Mole Ratio "(18) لمعرفة النسب التركيبي لناتج 4-AAP مع الثيامين باستخدام محاليل مخففة من الكاشف والمركب المدروس وبتركيز 1.0×10 -3 مولاري. النتائج الموضحة في الثكل (4) والثكل (5) تؤكد ان الناتج يتكون بنسبة 1:1 (الثيامين : AAP) لكلا الطريقتين. وتم احتساب ثابت استقرار النواتج المتكونة بنسبة 1:1 وبشكل منفصل بتطبيق القانون الاتي: $\mathrm{K}_{\mathrm{st}}=\frac{1-\alpha}{\alpha^{2} \mathrm{C}}$

حيث C هي تركيز المعقد بوحدة (مول/ لتر)، و $\alpha$ هي درجـة التفكك و كابت الاستقرار، بلـغ معدل ثابت الاستقرار للمعقد

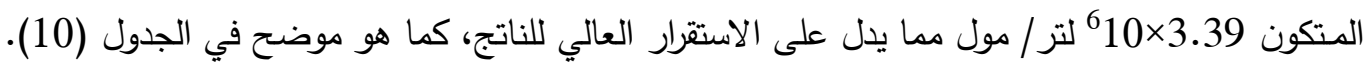

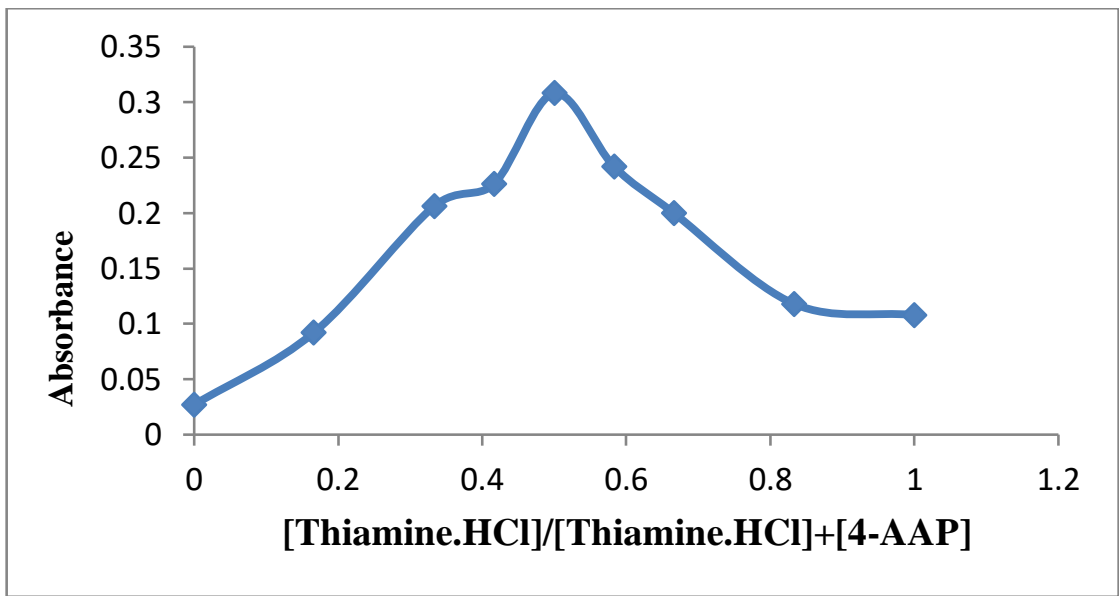

الثكل (4) رسم التغييرات المستمـرة لمعقد الثيامين مع 4-امينوانتي بايرين

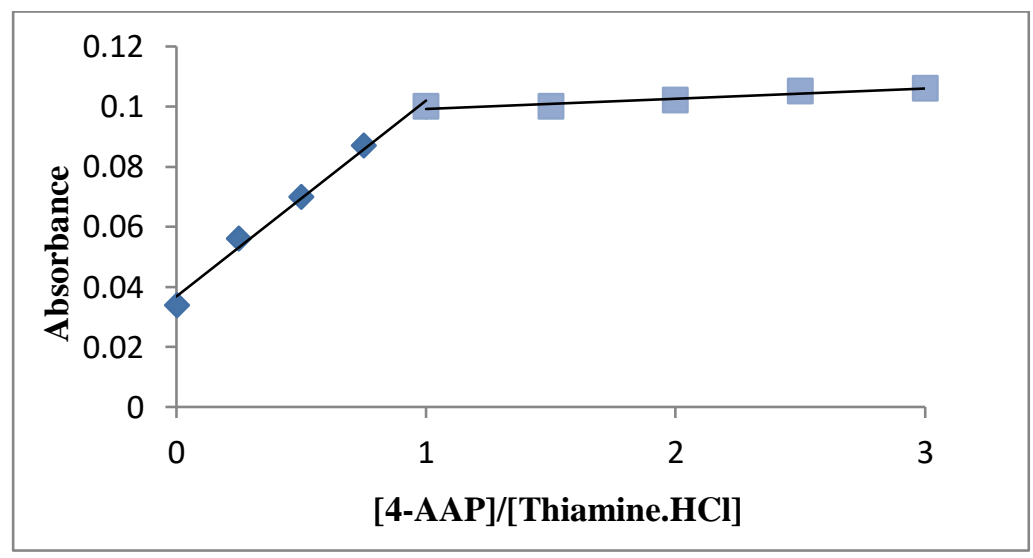

الثكل (5) رسم النسب المولية لمعقد الثيامين مع 4-امينوانتي بايرين 
الجدول (10) ثابت استقرار المعقد مع الكاشف 4-امينوانتي بايرين

\begin{tabular}{|c|c|c|c|c|c|}
\hline \multirow{2}{*}{ Compound } & \multirow{2}{*}{$\begin{array}{c}\text { Conc. } \\
\left(\mathrm{mol} . \mathrm{l}^{-1}\right)\end{array}$} & \multicolumn{2}{|c|}{ Absorbance } & \multirow{2}{*}{$\alpha$} & \multirow{2}{*}{$\begin{array}{l}\text { Average } \mathbf{K}_{\mathrm{s}} \\
\quad\left(\mathbf{l} \mathrm{mol}^{-1}\right)\end{array}$} \\
\hline & & As & Am & & \\
\hline \multirow{3}{*}{$\begin{array}{c}\text { Thiamine } \\
\text { hydrochloride }\end{array}$} & $2 \times 10^{-6}$ & 0.053 & 0.086 & 0.384 & \multirow{3}{*}{$3.39 \times 10^{6}$} \\
\hline & $4 \times 10^{-6}$ & 0.160 & 0.198 & 0.192 & \\
\hline & $6 \times 10^{-6}$ & 0.192 & 0.247 & 0.223 & \\
\hline
\end{tabular}

دراسة الـمتداخـلات

Glucose, Lactose, Sucrose, ) درس عدد من المتداخلات والمتوقع تواجدها في المستحضرات الصيدلانية والمتمثلة بـ Starch, Talc, Mg - stearte في امتصاص (4 مايكروغرام/ مللتر) من الثيامين، فقد اضيفت كميات مختلفة من المتداخلات الى المحاليل وقيس الامتصاص تحت الظروف المثلى عند 335 نانوميتر • تشير النتائج ادناه الى انتقائية الطريقة لعدم وجود تداخل من قبل المواد السواغ المضافة.

الجدول (11) تأثير المتداخلات

\begin{tabular}{|c|c|c|c|c|}
\hline \multirow[t]{2}{*}{ Foreign compound } & \multicolumn{4}{|c|}{$\begin{array}{c}\text { Recovery }(\%) \text { of } 4 \mu \mathrm{g} / \mathrm{ml} \text { of Thiamine } . \mathrm{HCl} \text { per } \\
\mu \mathrm{g} / \mathrm{ml} \text { Foreign added }\end{array}$} \\
\hline & 100 & 500 & 750 & 1000 \\
\hline Glucose & 101.9 & 99.0 & 101.0 & 98.6 \\
\hline Lactose & 96.0 & 96.9 & 101.4 & 101.5 \\
\hline Sucrose & 101.0 & 102.0 & 104.1 & 101.2 \\
\hline Starch & 95.6 & 97.8 & 101.3 & 103.0 \\
\hline Talc & 96.5 & 102.2 & 104.0 & 99.9 \\
\hline Mg-stearate & 99.4 & 103.1 & 98.7 & 102.0 \\
\hline
\end{tabular}

التفاعل الكيميـائي المقترح لوحظ من خلال التركيب الكيميائي لهيدروكلوريد الثيامين احتوائه على مجموعة الامين الاروماتية ومن خلال دراسة طبيعة الناتج المتكون بطريقتي جوب والنسبة المولية اثتتت النتيجة بان الارتباط يكون بنسبة 1:1 بوجود العامل المؤكسد في الوسط القاعدي كما هو الهوني موضتح في الميكانيكية المقترحة ادناه. 

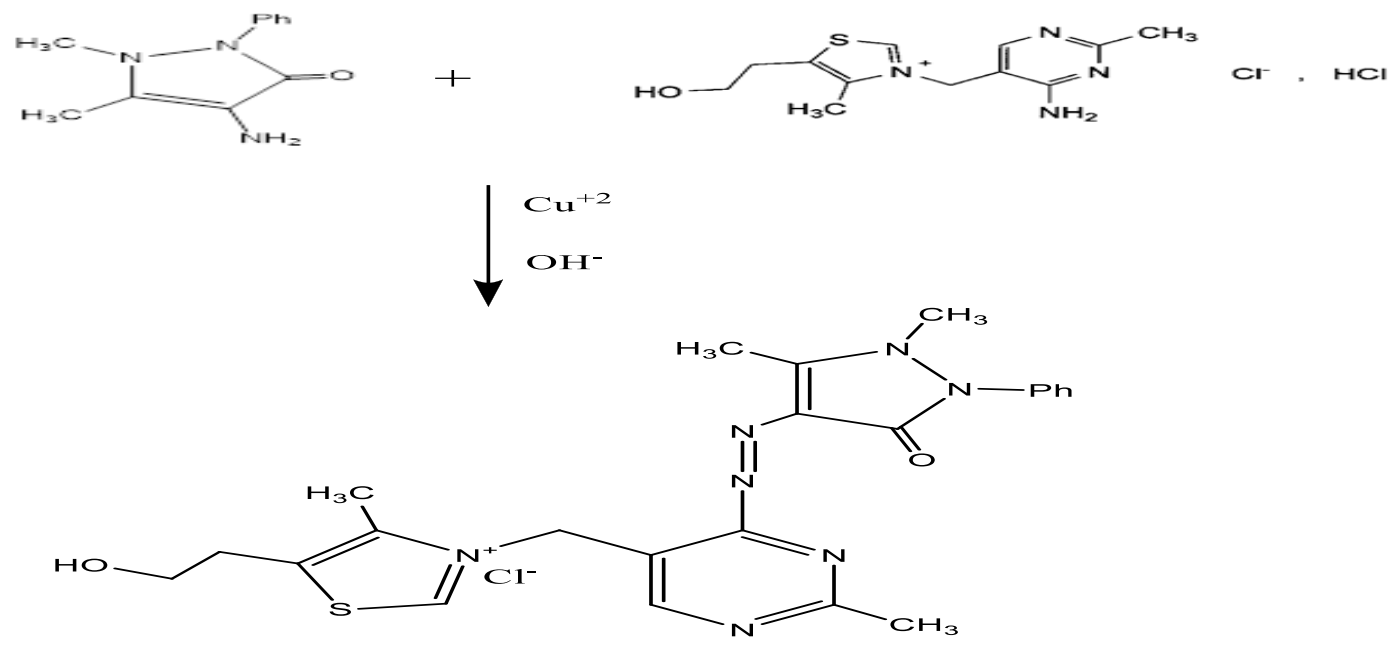

تحليل اقراص المستحضر الصيدلاني تم وزن خمسة اقراص من المستحضر الدوائي ( Neurorubine ) ثم طحنت ومزجت بشكل جيذ ومن ثم وزن من المسحوق مايكافى قرص واحد (200 ملغم)، ثم اذيبت في كمية قليلة من الماء المقطر ، بعدها رشح المحلول وأكمل الحجم الى 100 مللتر بالماء المقطر • وحضر منه محلول بتركيز 100 جزء لكل مليون ثم اخذ حجوم مختلفة منه للحصول على تراكيز (1.0 و 4.0 و 8.0 مايكروغرام/ مللتر ، تم ايجاد التركيز باستخدام المنحنى القياسي للثيامين بصورته النقيـة وادرجت النتائج في الجدول ادناه، اذ تبين ان الطريقة المتبعة ذات دقـة عالية ومتفقـة على نحو جيد مـع المحتوى الاصيل للثيامين في المستحضر الصـيدلاني.

\begin{tabular}{|c|c|c|c|c|c|}
\hline $\begin{array}{c}\text { Pharmaceutical } \\
\text { preparation }\end{array}$ & $\begin{array}{l}\text { Certified } \\
\text { value }\end{array}$ & $\begin{array}{l}\text { Amount } \\
\text { present } \\
\text { (ppm) }\end{array}$ & $\begin{array}{c}\text { Drug } \\
\text { content } \\
\text { found* }\end{array}$ & $\begin{array}{c}\text { Recovery* } \\
(\%)\end{array}$ & $\begin{array}{c}\text { Average } \\
\text { recovery } \\
(\%)\end{array}$ \\
\hline \multirow{3}{*}{$\begin{array}{l}\text { Neurorubine } \\
\text { Tablets } \\
\text { Switzerland }\end{array}$} & \multirow{3}{*}{$200 \mathrm{mg}$} & 1 & 199.02 & 99.51 & \multirow{3}{*}{100.56} \\
\hline & & 4 & 203.28 & 101.64 & \\
\hline & & 8 & 201.08 & 100.54 & \\
\hline
\end{tabular}

*Average of five determinations

تقييـم نتـائج الطريقة المقترحة مـع طريقة الاضافة القياسية لاثبات كفاءة الطريقة المقترحة ونجاحها في التقدير وخلوها من تأثير تداخلات الاضافات، طبقت طريقة الاضافة القياسية على النموذج الدوائي للثيامين لعدم توفر مستلزمات الطريقة القياسيـة المعتمدة في دستور الادويـة البريطاني(19) لتقدير الثيامين هيدروكلوريد، اذ يمكن الاستدلال من النتائج المبينة في الثكل (6) و الجدول (13) ان الطريقة المستحصلة متفقة مع الطريقة المقترحة مما يدل على أن الطريقة ذات انتقائية جيدة. 


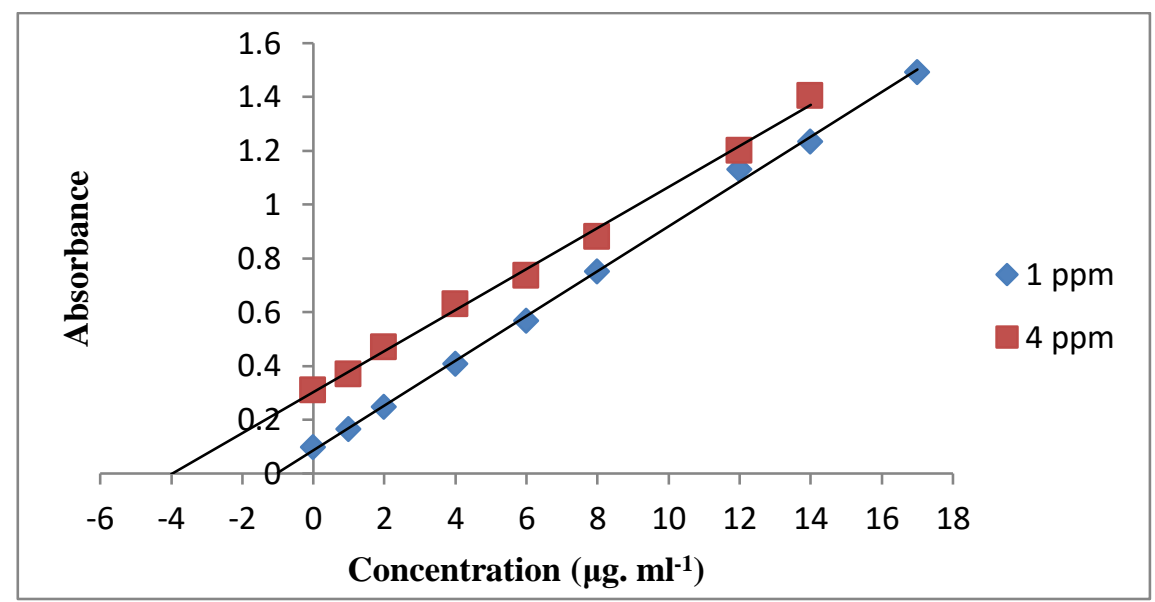

الشكل (6) منحنى الاضافـة القياسيـة لتقدير مستحضر الثيامين في الاقراص السويـرية من شركة (Acino Pharma)

الجدول (13) مقـارنة دقة الطريـقة المقترحة لتقدير المركب الدوائي في المستحضر الصيدلاني مع طريقة الاضافة القياسية

\begin{tabular}{|c|c|c|c|c|c|}
\hline \multirow{2}{*}{$\begin{array}{c}\text { Pharmaceutical } \\
\text { preparation }\end{array}$} & \multirow{2}{*}{$\begin{array}{c}\text { Certified } \\
\text { value }(\mathbf{m g})\end{array}$} & \multirow{2}{*}{$\begin{array}{c}\text { Amount } \\
\text { present } \\
\text { (ppm) }\end{array}$} & \multicolumn{2}{|c|}{ Drug content found (mg) } & \multirow{2}{*}{$\begin{array}{c}\text { Recovery }(\%) \text { of } \\
\text { standard } \\
\text { addition } \\
\text { procedure }\end{array}$} \\
\hline & & & $\begin{array}{l}\text { Present } \\
\text { method* }\end{array}$ & $\begin{array}{c}\text { Standard } \\
\text { addition } \\
\text { procedure }\end{array}$ & \\
\hline $\begin{array}{l}\text { Neurorubine } \\
\text { Tablets }\end{array}$ & \multirow{2}{*}{200} & 1 & 199.02 & 199.79 & 100.38 \\
\hline $\begin{array}{l}\text { Switzerland - } \\
\text { Acino Pharma }\end{array}$ & & 4 & 203.28 & 202.50 & 99.61 \\
\hline
\end{tabular}

*Average of five determinations

طورت طريقة طيفية لتقدير الثيامين في المحلول المائي مـع 4-امينوانتي بايرين وكبريتات النحاس المائية كعامل مؤكسد في الوسط القاعدي اعتماداً على تفـاعل الاقتران التأكسدي وقياس الناتج عند طول موجي 335 نانوميتر، امكن تقدير كميات متتاهيـة في الصغـر (0.2- 18) مايكروغرام/ مللتر وبامتصاصية مولاريـة 26410.59 لتر/ مول. سم وبدقة جيدة، بلغت كمية الاسترجاعية

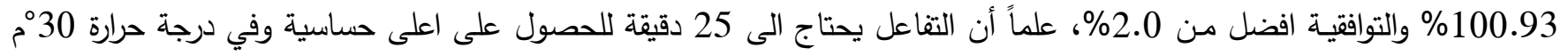
وقد امكن تطبيق الطريقة بنجاح على المستحضر الصيدلاني (Neurorubine). كما وجد من خلال دراسة طبيعة المعقد ان المعقد يتكون بنسبة 1:1، وتميزت الطريقة المطورة بالحساسية والسهولة وعدم الحاجة الى استخلاص مسبق. 


\section{References}

2. M. E. Webb, A. Marquet, R. R. Mendel, F Rebeille and A. G. Smith, "Elucidating biosynthetic pathways for vitamins and cofactors”. Nat. Prod. Rep.24(5): 988-1008, (2007).

3. S. S Barbara, Journal of Analytical Chemistry, 68,218-222, (2013).

4. H. M. A. Al-Guhashi, M.Sc. Thesis, Mosul University, (2019).

5. H. S. Al-Ward and S. Z. Hussein, International Journal of Pharmaceutical Sciences and Research, 7(10), 3995-03, (2016).

6. S.T. Abdel Rahman, A. A. Elbashir, M. El-Mukhtar and M. M. Ibrahim, Bio analytical Techniques, 2, $1-6,(2015)$.

7. N. H. Shekho, B. A. Abed Al-Hadi and L. A. Sarsam, Rafidain Journal of Sciences, 24, 60-73, (2013).

8. A. B. M. Al-Khalily, M.Sc. Thesis, University of Karbala, (2010).

9. N. R. Ahmad and F. K. Omar, Baghdad Science Journal, 16(4), 898-902, (2019).

10. S.T. Abdel Rahman, A. A. Elbashir, M. El-Mukhtar and M. M. Ibrahim, Journal of Analytical and Pharmaceutical Research, 2, 1-6, (2016).

11. B. A. Abed Al-Hadi, Tikrit Journal of Pure Science 24, (1), 74-81, (2019).

12. N. R. Ahmad and W. A. Al-Qazzaz, Rafidain Journal of Sciences, 28(2), 146-151, (2019).

13. H. Soni, A. K Singhai, K. Mishra and S. Sharma, International Journal of Pharmaceutical Sciences and Research, 3, 2163-2167, (2012).

14. M. L. Marszałł, A. Lebiedzińska, W. Czarnowski and P. Szeferb, J Chromatogr A, 1094(1-2), 9198, (2005).

15. S. Antakli, N. Sarkees and T. Sarraf, International Journal of Pharmacy and Pharmaceutical Sciences, 7(6), 219-224, (2015).

16. J. Tan, R. Li and Z. T. Jiang, Analytical Methods, 3,1568-1573, (2011). 
17. I. G. David, M. A. Florea, O. G. Cracea, D. E. Popa, M. Buleandra and E. E. Iorgulescu et al, Chemical Papers. 69(7), 901-910, (2015).

18. H. H. Willard, L. M. Merrit and J. A. Dean, "Instrumental methods of analysis", 5th ed., D. van Nostr and company NewYork, 121 (1974).

19. L. G. Hargis, “Anaytical Chemistry” Prentice-Hall.Inc., New Jersey, 424-427 (1988).

20. British Pharmacopoeia 2013. London, Stationery Office, 7th Edition. 